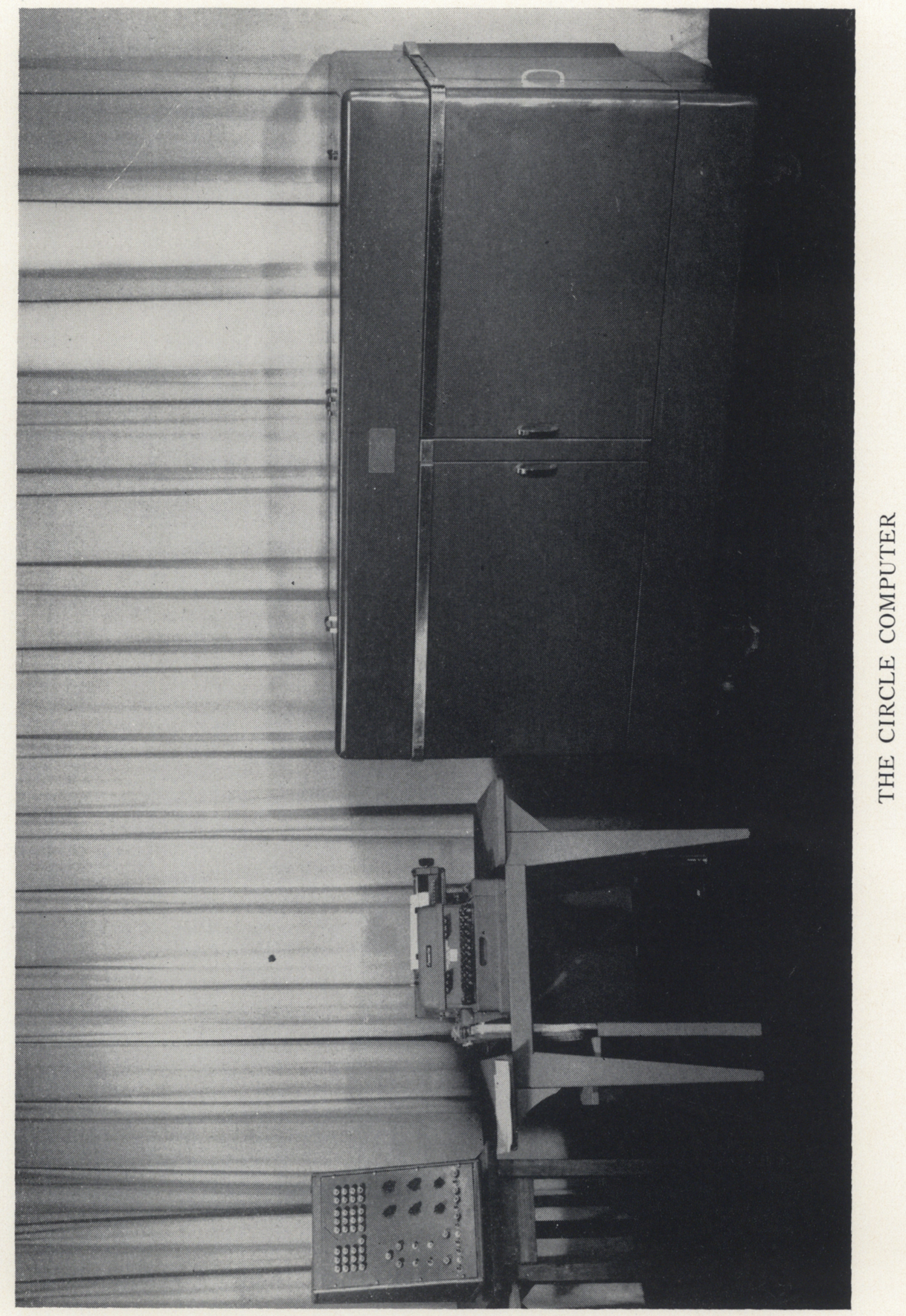




\section{Computing Eigenvalues and Eigenvectors of a Symmetric Matrix on the ILLIAC}

One of the programs in the library of programs for the University of Illinois' electronic digital computer, known as the ILLIAC, is a program for finding the eigenvalues and eigenvectors of a symmetric matrix. The iterative method used is the rotation of axes method discussed by H. H. GoldstinE ${ }^{1}$ in an unpublished paper and referred to by TAUSSKY \& TODD ${ }^{2}$ as JACOBI's method. ${ }^{3}$ It consists essentially of performing a sequence of orthogonal transformations on the matrix, where each transformation is designed to reduce a selected off-diagonal element to zero. GoLDSTINE ${ }^{1}$ shows that the sum of the squares of the off-diagonal elements is reduced, during a single transformation, by the amount $2 a^{2}{ }_{j k}\left(a_{j k}\right.$ is the element reduced to zero by the transformation) and that the process produces a sequence of matrices whose limit is a diagonal matrix. His expression for an upper bound on the number of transformations required to diagonalize an $n$th order matrix is $\left[\ln \left(t_{0} / t_{i}\right)\right]\left(n^{2}-n\right) / 2$, where $t_{0}$ is the sum of the squares of the off-diagonal elements of the original matrix and $t_{i}$ is the same quantity after the $i$ th transformation. Here it is assumed that $a_{j k}$ is always greater than the average off-diagonal element in absolute value. Our results so far indicate that this bound is from ten to twenty times greater than the number actually required by our program. The eigenvectors are obtained by multiplying together the orthogonal matrices used in the successive transformations.

When the eigenvalues are not close, and the element $a_{j k}$ is small, reduction of $a_{j k}$ to 0 leaves the other elements unchanged in the first approximation. For the angle of rotation is given by the relation

$$
\tan 2 \phi=2 a_{j k} /\left(a_{j j}-a_{k k}\right) .
$$

When the off-diagonal elements are very small $\phi$ is of the order of $a_{j k}$ $\left(a_{j j} \neq a_{k k}\right)$. Now off-diagonal elements are transformed by

$$
\begin{array}{r}
a_{r j}=a_{r j} \cos \phi+a_{r k} \sin \phi=a_{r j}\left(1-\phi^{2} / 2 !+\cdots\right) \\
\begin{aligned}
a_{r k}=-a_{r k}\left(\phi-\phi^{3} / 3 !+\cdots\right) \\
\\
+a_{r k}\left(1-\phi^{2} / 2 !+\cdots\right)
\end{aligned}
\end{array}
$$

and hence are unchanged if second order terms are neglected. Thus one sweep through the off-diagonal elements reduces them to zero (up to terms of second order).

The purpose of this paper is to display some results of an investigation into the relative merits of

(1) two approaches to the problem of how to select the off-diagonal element $a_{j k}$ mentioned above, and

(2) two approaches to the problem of when to apply the convergence test so as to terminate the process after convergence.

The two approaches mentioned in (1) are

(a) to select the off-diagonal elements in sequence along successive rows of the matrix, and

(b) to select the largest off-diagonal element each time.

The two approaches mentioned in (2) are

(c) to apply the convergence test after each transformation, and 
(d) to apply the convergence test after each group of $\left(n^{2}-n\right) / 2$ transformations, where $n$ is the order of the matrix. The convergence test used was a test of the size of $t_{i}$ using double precision.

Method (a) is the simplest to program for an electronic computer but will require more transformations for convergence than method (b). Thus the accumulated round-off errors should be less using method (b). Method (c) enables one to terminate the process as soon as the process converges, but requires many applications of the test. Method (d) applies the test only after going through the off-diagonal elements once. This means that overiterating will result and in the extreme case $\left(n^{2}-n-2\right) / 2$ unnecessary transformations will be performed.

The library program mentioned in the first paragraph is called program 42. It uses methods (a) and (d) and requires a total of 190 storage locations in the memory. Two modifications of this program have been written which are slightly longer. Program 42A uses methods (a) and (c) and program 42B uses methods (b) and (c). Tables 1-5 display the results obtained using these three programs on seven matrices of each of the orders $20,16,12,8$, and 4 . They contain

A. The time required to diagonalize each matrix,

B. The number of orthogonal transformations required, and

C. An indication of the accuracy.

Tables 6-9 contain the eigenvalues of the thirty-five matrices. In order to conserve space only five decimal places are included.

The time referred to in $\mathrm{A}$ is merely the computation time and does not include the time required for input or output of data. The accuracy of the process (item $\mathrm{C}$ above) is determined by forming the sum of the squares of the components of the $n$ residual vectors,

$$
r_{i}=A x_{i}-\pi_{i} x_{i} \quad i=1,2, \cdots, n
$$

where $x_{i}$ and $\pi_{i}$ are the eigenvectors and eigenvalues, respectively, of A. This sum of squares is small and is scaled by $2^{30}$ before being printed.

Of the thirty-five matrices used in this investigation five were correlation matrices which were available (numbers $1,8,15,22$, and 29) and the remaining thirty were matrices generated by the machine. The method employed to generate the elements of these matrices was to square a number and use the middle digits of the product. Each new number then was used to generate the following number. The computation times for diagonalizing the five correlation matrices are slightly longer than those for the machine generated matrices due to the fact that certain changes were made in the ILLIAC, just after the five correlation matrices were diagonalized, which increased the speed of certain arithmetic operations.

Several conclusions can be drawn from an inspection of the results. The simplest program (number 42) using methods (a) and (d) was the fastest despite the fact that it over-iterated. However program 42B, using method (b), was in general the most accurate in the sense that the sum of squares of residuals was smallest. Obviously, the method (d) is superior to method (c). Program 42 never required more than seven sweeps through the off- 
diagonal elements, i.e., no more than $7\left(n^{2}-n\right) / 2$ transformations were required for convergence. It appears that method (a) required about one and one-half times as many transformations as method (b).

\begin{tabular}{|c|c|c|c|c|}
\hline \multirow[b]{2}{*}{ Matrix } & \multicolumn{2}{|c|}{ Time } & \multirow{2}{*}{$\begin{array}{l}\text { Number of } \\
\text { Transformations }\end{array}$} & \multirow{3}{*}{$\frac{n=20}{2^{30} \Sigma r_{i j}^{2}}$} \\
\hline & Min. & Sec. & & \\
\hline \multicolumn{4}{|c|}{ PROGRAM 42} & \\
\hline $\begin{array}{l}1 \\
2 \\
3 \\
4 \\
5 \\
6 \\
7\end{array}$ & $\begin{array}{l}6 \\
5 \\
5 \\
5 \\
5 \\
5 \\
5\end{array}$ & $\begin{array}{r}39 \\
57 \\
56 \\
56 \\
6 \\
56 \\
6\end{array}$ & $\begin{array}{l}1330 \\
1330 \\
1330 \\
1330 \\
1140 \\
1330 \\
1140\end{array}$ & $\begin{array}{l}.00345 \\
.00331 \\
.00286 \\
.00310 \\
.00251 \\
.00305 \\
.00296\end{array}$ \\
\hline \multicolumn{5}{|c|}{ PROGRAM 42A } \\
\hline $\begin{array}{l}1 \\
2 \\
3 \\
4 \\
5 \\
6 \\
7\end{array}$ & $\begin{array}{l}15 \\
14 \\
14 \\
14 \\
13 \\
14 \\
13\end{array}$ & $\begin{array}{l}18 \\
25 \\
27 \\
17 \\
32 \\
23 \\
56\end{array}$ & $\begin{array}{l}1154 \\
1161 \\
1164 \\
1149 \\
1087 \\
1159 \\
1122\end{array}$ & $\begin{array}{l}.00272 \\
.00279 \\
.00282 \\
.00326 \\
.00230 \\
.00314 \\
.00263\end{array}$ \\
\hline \multicolumn{5}{|c|}{ PROGRAM 42B } \\
\hline $\begin{array}{l}1 \\
2 \\
3 \\
4 \\
5 \\
6 \\
7\end{array}$ & $\begin{array}{l}19 \\
19 \\
19 \\
19 \\
19 \\
19 \\
19\end{array}$ & $\begin{array}{l}34 \\
19 \\
32 \\
32 \\
18 \\
17 \\
13\end{array}$ & $\begin{array}{l}683 \\
685 \\
692 \\
692 \\
684 \\
684 \\
681\end{array}$ & $\begin{array}{l}.00145 \\
.00138 \\
.00131 \\
.00167 \\
.00148 \\
.00141 \\
.00165\end{array}$ \\
\hline
\end{tabular}

TABLE 1

\begin{tabular}{|c|c|c|c|c|}
\hline \multirow[b]{2}{*}{ Matrix } & \multicolumn{2}{|c|}{ Time } & \multirow{2}{*}{$\begin{array}{c}\text { Number of } \\
\text { Transformations }\end{array}$} & \multirow{2}{*}{$\frac{n=16}{2^{30} \Sigma r_{i j}^{2}}$} \\
\hline & Min. & $\begin{array}{r}\text { Sec. } \\
\text { PR }\end{array}$ & & \\
\hline $\begin{array}{r}8 \\
9 \\
10 \\
11 \\
12 \\
13 \\
14\end{array}$ & $\begin{array}{l}3 \\
3 \\
3 \\
3 \\
2 \\
2 \\
2\end{array}$ & $\begin{array}{r}28 \\
6 \\
6 \\
5 \\
39 \\
39 \\
39\end{array}$ & $\begin{array}{l}840 \\
840 \\
840 \\
840 \\
720 \\
720 \\
720\end{array}$ & $\begin{array}{l}.00191 \\
.00163 \\
.00149 \\
.00142 \\
.00131 \\
.00104 \\
.00152\end{array}$ \\
\hline \multicolumn{5}{|c|}{ PROGRAM 42A } \\
\hline $\begin{array}{r}8 \\
9 \\
10 \\
11 \\
12 \\
13 \\
14\end{array}$ & $\begin{array}{l}6 \\
6 \\
6 \\
6 \\
5 \\
5 \\
5 \\
5\end{array}$ & $\begin{array}{l}54 \\
23 \\
23 \\
21 \\
53 \\
50 \\
37\end{array}$ & $\begin{array}{l}726 \\
724 \\
725 \\
721 \\
667 \\
663 \\
637\end{array}$ & $\begin{array}{l}.00163 \\
.00126 \\
.00106 \\
.00133 \\
.00126 \\
.00106 \\
.00154\end{array}$ \\
\hline \multicolumn{5}{|c|}{ PROGRAM 42B } \\
\hline $\begin{array}{r}8 \\
9 \\
10 \\
11 \\
12 \\
13 \\
14\end{array}$ & $\begin{array}{l}8 \\
8 \\
7 \\
8 \\
7 \\
7 \\
7\end{array}$ & $\begin{array}{r}10 \\
0 \\
53 \\
4 \\
47 \\
54 \\
57\end{array}$ & $\begin{array}{l}425 \\
432 \\
426 \\
436 \\
421 \\
427 \\
430\end{array}$ & $\begin{array}{l}.00063 \\
.00066 \\
.00061 \\
.00070 \\
.00063 \\
.00072 \\
.00059\end{array}$ \\
\hline
\end{tabular}

TABLE 2 


\begin{tabular}{|c|c|c|c|c|}
\hline \multirow[b]{2}{*}{ Matrix } & \multicolumn{2}{|c|}{ Time } & \multirow{2}{*}{$\begin{array}{l}\text { Number of } \\
\text { Transformations }\end{array}$} & \multirow{2}{*}{$\frac{n=12}{2^{20} \Sigma r_{i j}^{2}}$} \\
\hline & Min. & Sec. & & \\
\hline \multicolumn{5}{|c|}{ PROGRAM 42} \\
\hline $\begin{array}{l}15 \\
16 \\
17 \\
18 \\
19 \\
20 \\
21\end{array}$ & $\begin{array}{l}1 \\
1 \\
1 \\
1 \\
1 \\
1 \\
1\end{array}$ & $\begin{array}{r}17 \\
9 \\
9 \\
9 \\
9 \\
9 \\
9\end{array}$ & $\begin{array}{r}396 \\
396 \\
396 \\
396 \\
396 \\
396 \\
396\end{array}$ & $\begin{array}{l}.000600 \\
.000591 \\
.000447 \\
.000450 \\
.000502 \\
.000579 \\
.000600\end{array}$ \\
\hline \multicolumn{5}{|c|}{ PROGRAM 42A } \\
\hline $\begin{array}{l}15 \\
16 \\
17 \\
18 \\
19 \\
20 \\
21\end{array}$ & $\begin{array}{l}2 \\
1 \\
1 \\
1 \\
2 \\
2 \\
2\end{array}$ & $\begin{array}{r}10 \\
59 \\
58 \\
58 \\
2 \\
2 \\
1\end{array}$ & $\begin{array}{l}344 \\
340 \\
338 \\
339 \\
351 \\
351 \\
347\end{array}$ & $\begin{array}{l}.000681 \\
.000492 \\
.000506 \\
.000369 \\
.000407 \\
.000501 \\
.000631\end{array}$ \\
\hline \multicolumn{5}{|c|}{ PROGRAM 42B } \\
\hline $\begin{array}{l}15 \\
16 \\
17 \\
18 \\
19 \\
20 \\
21\end{array}$ & $\begin{array}{l}2 \\
2 \\
2 \\
2 \\
2 \\
2 \\
2\end{array}$ & $\begin{array}{l}33 \\
41 \\
33 \\
35 \\
25 \\
32 \\
39\end{array}$ & $\begin{array}{l}217 \\
239 \\
228 \\
230 \\
216 \\
227 \\
236\end{array}$ & $\begin{array}{l}.000233 \\
.000307 \\
.000277 \\
.000233 \\
.000256 \\
.000257 \\
.000232\end{array}$ \\
\hline
\end{tabular}

TABLE 3

\begin{tabular}{|c|c|c|c|c|}
\hline \multirow[b]{2}{*}{ Matrix } & \multicolumn{2}{|c|}{ Time } & \multirow{2}{*}{$\begin{array}{c}\text { Number of } \\
\text { Transformations }\end{array}$} & \multirow{2}{*}{$\frac{n=8}{2^{50} \Sigma r_{i j}^{2}}$} \\
\hline & Min. & Sec. & & \\
\hline \multicolumn{5}{|c|}{ PROGRAM 42} \\
\hline $\begin{array}{l}22 \\
23 \\
24 \\
25 \\
26 \\
27 \\
28\end{array}$ & & $\begin{array}{l}20 \\
18 \\
18 \\
18 \\
18 \\
22 \\
18\end{array}$ & $\begin{array}{r}140 \\
140 \\
140 \\
140 \\
140 \\
168 \\
140\end{array}$ & $\begin{array}{l}.000125 \\
.000134 \\
.000119 \\
.000118 \\
.000111 \\
.000165 \\
.000102\end{array}$ \\
\hline \multicolumn{5}{|c|}{ PROGRAM 42A } \\
\hline $\begin{array}{l}22 \\
23 \\
24 \\
25 \\
26 \\
27 \\
28\end{array}$ & & $\begin{array}{l}28 \\
28 \\
26 \\
25 \\
28 \\
29 \\
28\end{array}$ & $\begin{array}{r}126 \\
135 \\
129 \\
121 \\
134 \\
133 \\
143 \\
135\end{array}$ & $\begin{array}{l}.000160 \\
.000113 \\
.000112 \\
.000106 \\
.000088 \\
.000208 \\
.000102\end{array}$ \\
\hline \multicolumn{5}{|c|}{ PROGRAM 42B } \\
\hline $\begin{array}{l}22 \\
23 \\
24 \\
25 \\
26 \\
27 \\
28\end{array}$ & & $\begin{array}{l}35 \\
32 \\
30 \\
32 \\
32 \\
34 \\
34\end{array}$ & $\begin{array}{l}97 \\
91 \\
88 \\
94 \\
93 \\
96 \\
97\end{array}$ & $\begin{array}{l}.000072 \\
.000047 \\
.000085 \\
.000063 \\
.000056 \\
.000043 \\
.000081\end{array}$ \\
\hline
\end{tabular}

TABLE 4 


\begin{tabular}{|c|c|c|c|c|}
\hline \multirow{2}{*}{ Matrix } & \multicolumn{2}{|c|}{ Time } & \multirow{2}{*}{$\begin{array}{c}\text { Number of } \\
\text { Transformations }\end{array}$} & \multirow{2}{*}{$\frac{n=4}{2^{30} \Sigma r_{i j}^{2}}$} \\
\hline & Min. & Sec. & & \\
\hline \multicolumn{5}{|c|}{ PROGRAM 42} \\
\hline $\begin{array}{l}29 \\
30 \\
31 \\
32 \\
33 \\
34 \\
35\end{array}$ & & $\begin{array}{l}2.5 \\
2.4 \\
3.0 \\
2.4 \\
2.3 \\
2.4 \\
2.4\end{array}$ & $\begin{array}{l}24 \\
24 \\
30 \\
24 \\
24 \\
24 \\
24\end{array}$ & $\begin{array}{l}.0000212 \\
.000009 \\
.000007 \\
.000007 \\
.000013 \\
.000011 \\
.000008\end{array}$ \\
\hline
\end{tabular}

PROGRAM 42A

2.4

19

2.320

2.925

$2.1 \quad 19$

$2.3 \quad 20$

$2.4 \quad 22$

2.422

.0000138

.0000056

.0000113

.0000029

.0000093

.0000078

PROGRAM 42B

29
30
31
32
33
34
35

2.4
2.3
2.4
2.4
2.4
2.4
2.4

17

16

16

16

17

16

.0000143

TABLE 5

.0000055

.0000051

.0000053

.0000030

.0000042

.0000067

.0000090

EIGENVALUES

\begin{tabular}{|c|c|c|c|c|c|c|}
\hline 1 & 2 & 3 & 4 & 5 & 6 & 7 \\
\hline $\begin{array}{r}+.72951 \\
-.75242 \\
-.54106 \\
+.41564 \\
-.58256 \\
-.28102 \\
+.52031 \\
+.58046 \\
-.41469 \\
-.29046 \\
-.33468 \\
+.54923 \\
+.02789 \\
+.18720 \\
.18534 \\
+.13149 \\
+.29696 \\
+.05071 \\
+.13306 \\
-.05810\end{array}$ & $\begin{array}{r}+.70548 \\
-.54004 \\
-.50730 \\
-.23097 \\
-.64287 \\
+.58653 \\
+.26919 \\
+.10555 \\
+.29675 \\
+.52572 \\
+.37614 \\
.43715 \\
+.48254 \\
+.35102 \\
+.16190 \\
-.24689 \\
+.06814 \\
+.03994 \\
+.17470 \\
+.21225\end{array}$ & $\begin{array}{r}+.61214 \\
-.38161 \\
+.45455 \\
-.72650 \\
-.53514 \\
-.43796 \\
+.75447 \\
+.55998 \\
.30252 \\
+.12832 \\
+.27717 \\
.07906 \\
+.30587 \\
+.48978 \\
+.21471 \\
-.23067 \\
-.21203 \\
+.23868 \\
.03577 \\
+.02712\end{array}$ & $\begin{array}{r}-.60855 \\
-.52812 \\
-.43533 \\
+. .78575 \\
+.46933 \\
. .64543 \\
+.27829 \\
+.71874 \\
-.32482 \\
-.22613 \\
+.59033 \\
+.35744 \\
+.07084 \\
+.21038 \\
.11323 \\
+.09255 \\
+.02895 \\
-.27643 \\
-.06418 \\
-.16923\end{array}$ & $\begin{array}{r}+.34710 \\
+.56507 \\
. .74842 \\
+.54166 \\
+.61324 \\
-.27147 \\
-.49548 \\
-.52582 \\
+.52150 \\
+.45968 \\
-.34006 \\
. .10921 \\
+.29205 \\
.08419 \\
+.03378 \\
+.18037 \\
. .19826 \\
+.00225 \\
+.24630 \\
+.07460\end{array}$ & $\begin{array}{r}+.65011 \\
+.36704 \\
+. .73229 \\
+. .68674 \\
+.57402 \\
-.59379 \\
-.23771 \\
+.42814 \\
.49613 \\
+.24109 \\
+.34885 \\
.13801 \\
.46256 \\
+.27996 \\
+.05406 \\
+.20607 \\
-.41526 \\
-.28314 \\
-.05462 \\
-.18670\end{array}$ & $\begin{array}{r}+.55872 \\
-.71648 \\
+.60475 \\
-.33592 \\
-.53408 \\
+.69971 \\
+.36562 \\
+.37732 \\
-.60475 \\
+.29305 \\
.20574 \\
+.27637 \\
+.07809 \\
-.00028 \\
.12379 \\
+.41737 \\
+.20575 \\
.28980 \\
+.06703 \\
+.13132\end{array}$ \\
\hline
\end{tabular}

TABLE 6 
EIGENVALUES

\begin{tabular}{ccccccc}
8 & 9 & 10 & 11 & 12 & 13 & 14 \\
+.62833 & +.64790 & +.65658 & -.54089 & +.26411 & +.42710 & +.60556 \\
-.55535 & -.50376 & -.45344 & -.38215 & +.49948 & -.47585 & +.13948 \\
-.64081 & -.43511 & +.46503 & -.51589 & \pm .49670 & +.57891 & -.68004 \\
+.39019 & -.05532 & -.61847 & +.59733 & +.48355 & -.24169 & +.55796 \\
-.50721 & -.57457 & -.28238 & +.47695 & -.60610 & -.59440 & +.51185 \\
-.33063 & +.47444 & -.36903 & -.47087 & -.39261 & +.62438 & -.54204 \\
+.32001 & +.17786 & +.53729 & +.07674 & -.52051 & +.15495 & -.24080 \\
+.51316 & +.02452 & +.35702 & +.66319 & -.33774 & -.19197 & +.31760 \\
-.29364 & +.20854 & -.24871 & -.14883 & +.40512 & -.32898 & -.43488 \\
-.13233 & +.51314 & +.16411 & -.23124 & -.15733 & +.27840 & -.03264 \\
-.19180 & -.32012 & -.21132 & +.36210 & -.26548 & -.31017 & +.33752 \\
+.24308 & -.24645 & -.01296 & +.06539 & -.12244 & +.28416 & \pm .17560 \\
\hline .02683 & +.33257 & +.12396 & +.19461 & +.19613 & +.00504 & -.32918 \\
+.17290 & +.31621 & +.26874 & -.07243 & +.01189 & +.06621 & +.26982 \\
+.02344 & -.12027 & +.19060 & -.08335 & +.05150 & -.07676 & +.05161 \\
+.10861 & -.18881 & -.10576 & +.10039 & +.13602 & +.18048 & -.09026
\end{tabular}

TABLE 7

EIGENVALUES

\begin{tabular}{ccccccc}
15 & 16 & 17 & 18 & 19 & 20 & 21 \\
+.53175 & +.46872 & +.54439 & -.44956 & +.26122 & +.38378 & +.57151 \\
-.54732 & \pm .53186 & -.38947 & -.32509 & +.44123 & -.46303 & +.05192 \\
-.44538 & -.33823 & +.37032 & -.30085 & -.42957 & +.51761 & -.51638 \\
+.33602 & +.14008 & -.50660 & +.57480 & +.24992 & -.27931 & +.45467 \\
-.14756 & -.40958 & -.22885 & +.29884 & -.58716 & -.48722 & +.39733 \\
-.16680 & +.40960 & -.33077 & -.28068 & -.32414 & +.30840 & -.30701 \\
+.20161 & +.17662 & +.29323 & +.08503 & -.21857 & +.09502 & -.23531 \\
+.35719 & -.01891 & +.18885 & +.44081 & -.30374 & +.01101 & +.12498 \\
\hline .30020 & +.27312 & -.13267 & -.11625 & +.16273 & -.11119 & -.34457 \\
+.04760 & +.32131 & +.21655 & -.04640 & -.02549 & +.19796 & -.10689 \\
\hline .06842 & -.20311 & -.02120 & +.21271 & -.26387 & -.20737 & +.23361 \\
+.12093 & -.24408 & +.05281 & +.04666 & +.03641 & +.23940 & -.09563
\end{tabular}

TABLE 8

\section{EIGENVALUES}

\begin{tabular}{ccccccc}
22 & 23 & 24 & 25 & 26 & 27 & 28 \\
+.39745 & +.39005 & +.42526 & -.30457 & +.17009 & +.21844 & +.50218 \\
+.37863 & -.41997 & -.34751 & -.28376 & +.34870 & -.29635 & +.08990 \\
-.35548 & -.14337 & +.32709 & -.12749 & -.30897 & +.28240 & -.25758 \\
+.22612 & +.16712 & -.27489 & +.21208 & +.13591 & -.07422 & +.32270 \\
\hdashline .04768 & \pm .25903 & -.19429 & +.29716 & -.45210 & -.32743 & +.02900 \\
\hline .02841 & +.27762 & -.03599 & -.03213 & -.26639 & +.46710 & -.39965 \\
+.12765 & +.06557 & +.17299 & +.07477 & -.16227 & +.03887 & -.17113 \\
+.06131 & -.02717 & +.09796 & +.37703 & -.09466 & -.01919 & -.05480 \\
29 & 30 & & & & & \\
+.12263 & +.18964 & +.26306 & -.28179 & +.19975 & +.18424 & +.16572 \\
-.32853 & -.21833 & -.26037 & -.12093 & -.01266 & -.14856 & -.07177 \\
-.03726 & -.02269 & +.14103 & +.08960 & -.28262 & +.28318 & -.22650 \\
+.19951 & +.05038 & -.14230 & +.16096 & -.21660 & +.03009 & +.12923
\end{tabular}

TABLE 9

\footnotetext{
1 Institute for Advanced Study, Princeton, 1949.

20 . TAUSSKY \& J. TODD, "Systems of equations, matrices and determinants," Mathematics Magazine, v. 26, 1952, p. 71-88.

${ }^{3}$ C. G. J. JACOBI, "Ein leichtes Verfahren, die in der Theorie der Säkularstörungen vorkommenden Gleichungen numerisch aufzulösen," Jn. reine angew. Math., v. 30, 1846, p. 51-95.
}

University of Illinois

Urbana, Ill. 